\title{
Contents, Vol. 100, 1940
}

\section{Index}

Zum 100. Bande der Ophthalmologica - The 100th Volumeof Ophthalmologica - Au 100e Volume d'Ophthalmologica $\mathrm{V}$

Adamantiadis, B., et Djacos, C, Sur une famille atteinte dedystrophie granuleuse de la cornée (kératite de Græ-nouw) 33

Bangerter, A., Operationsmethode zum Verschluß von Netz-hautlöchern am hinteren Augenpol, im besonderen vonMaculalöchern 351

Bruckner, R., Ueber das säurelösliche Phosphat in den

Linsen verschiedener Tierarten 203

Bürki, E., Ueber Ultrakurzwellentherapie in der Ophthalmologie 43

Djacos, C, vide Adamantiadis, B.

Fischer F. P., Ueber Melanoidinbildung in der Linse. 150

Heßberg, R., Die Behandlung von Netzhautgefäßerkran-kungen, besonders der Venenthrombose, mit Röntgen-strahlen 74

Loewenstein, A., Lipoid (?) Droplets in the Episclera as a

Regular Change with Age 345

Lugossy, J., Chemotherapy of Paratrachoma .... 68

- The Problem of Chemotherapy in Trachoma . . 277

Macfadyen, J., Rupture of the Posterior Ciliary Arteries in

Eye Injuries by Blunt Objects 129

Mihályhegyi, G., Ueber Verbesserung der Sehschärfe und

Aenderung der Refraktion mittels Haftgläsern . . 193

Pick, L., Zur Behandlung der chronischen Konjunktivitis

und Blepharitis 208

Raáb, C. M., The Treatment of Eye Tuberculosis with aNew Dye Preparation. (With some facts relating tothe diagnosis of eye tuberculosis) (Tab. I-VI) $\quad$. $\quad 1$

Schlaeppi, V., De 1 influence des extraits post-hypophy-saires sur la pupille et la tension intraoculaire. (Etudeexpérimentale et clinique) . 321

Verhage, J. W. C, Der Nystagmus im niederländischenKohlenrevier. Wirtschaftliche Faktoren.

Photometri-sche Bestimmungen unter Tage 266

Weekers, R., Biochimie de la cornée normale et patholo-

gique 136

- Le role du Calcium dans le métabolisme hydrocarboné

du cristallin 257

Uebersichtsreferate - Reviews - Revues

Riddell, W. J. B., Developmental Abnormalities of the Eye.

(Period of Review July 1937-June 1939) . . “ 101, 159

Bruckner, A., Physiologische und psychologische Optik. 
(Berichtszeit 1937/1938)

$215,286,355$

Gesellschaftsberichte - Society Transactions - Sociétés

Sitzungsbericht der Niederländischen Ophthalmologischen Gesellschaft,

Utrecht, am 16. und 17. Dezember 1939243

Sitzungsbericht der 100. Versammlung der Niederländischen Ophthalmologischen Gesellschaft in Groningen am 5. Mai $1940 \ldots 372$

Diagnose und Therapie $\quad 120,179,253,316$

Necrologia

Anton Elschnig $\uparrow$. Von K. W. Ascher, Cincinnati

Felix Terrien (1872-1940). Par P. Bailliatt, Paris

Alfred Bielschowsky $\uparrow$. Von E. Th. von Brücke, Boston .... 318

B. Buchsprechungen - Books review - Livrcs nouveaux 183

256

Varia 127

192 